\title{
Lack of a bilateral projection of individual spinal neurons to the lateral reticular nucleus in the rat: a retrograde, non-fluorescent, double labeling study
}

\author{
S.K.E. Koekkoek, T.J.H. Ruigrok* \\ Department of Anatomy, Erasmus University Rotterdam, P.O. Box 1738, 3000 DR Rotterdam, The Netherlands
}

Received 28 June 1995; revised version received 13 September 1995; accepted 15 September 1995

\begin{abstract}
The projection of spinal neurons to the lateral reticular nucleus of the rat was investigated with a non-fluorescent double retrograde tracing technique. Either a gold-lectin tracer or cholera toxin-b-subunit was injected into the lateral reticular nucleus on each side of the brain. Retrogradely labeled neurons were encountered bilaterally throughout the spinal cord. Double labeled neurons, however, were seldom seen ( $<2 \%$ of the total number of labeled neurons) and those that were could, at least partly, be ascribed to inadvertent labeling of passing fibers. It is concluded that most spinoreticular neurons project to either the ipsi- or contralateral lateral reticular nucleus, suggesting that each side receives a unique spinal input.
\end{abstract}

Keywords: Cholera toxin-b-subunit; Gold lectin; Spinal cord; Cerebellum; Spinoreticular tract; Axon collaterals

The lateral reticular nucleus (LRt) is a relay nucleus between the spinal cord and the cerebellum. Unilateral LRt injections with retrograde tracers result in labeled spinal neurons, many of which are found bilaterally in similar laminae in the rat $[9,11,13]$ and in the cat [6]. Also, unilateral spinal cord injections of the anterograde tracer Phaseolus vulgaris leucoagglutinin (PHA-L) may result in bilateral projections to the LRt [11]. However, it remains to be determined whether individual neurons in the spinal cord project unilaterally (i.e. either ipsi- or contralaterally) or bilaterally to the LRt. Since information on this topic may be important to evaluate the function of spino-reticulo-cerebellar pathways in the control of (loco-) motor systems of the left and right side of the body, we have investigated a potential bilateral projection of spinal neurons to the LRt with a double retrograde tracing technique.

Surgery was performed on pentobarbital anaesthetized $(80 \mathrm{mg} / \mathrm{kg}$, i.p.) male Wistar rats $(n=5,200-250 \mathrm{~g})$ mounted in a stereotactic frame. Cholera toxin-b-subunit (CTb; low salt; List Biol. Lab., Campbell, CA) was iontophoretically injected ( $30 \mathrm{~min}, 2-4 \mu \mathrm{A})$ into the right LRt

\footnotetext{
* Corresponding author. Tel.: +31 104087296 ; fax: +31 104365780 .
}

through a glass micropipette (tip $10-15 \mu \mathrm{m}$ ). A retrogradely $[9,12]$ transported gold-lectin tracer, made up of $10 \mathrm{~nm}$ gold sol (Aurion, Wageningen, the Netherlands) coupled to a wheatgerm agglutinin and bovine serum albumin conjugate (WGA-BSA) [12], was pressure injected (100-200 nl) into the contralateral LRt. After 7-10 days, the rats were perfused ( $4 \%$ paraformaldehyde, $0.1 \%$ glutaraldehyde in phosphate buffer) and the brain and spinal cord were embedded in gelatin and cryoprotected. Transverse sections $(40 \mu \mathrm{m})$ were processed according to Ruigrok et al. [12]. Briefly, sections were incubated in goat anti-CTb (List Biol. Lab.) in Tris buffered saline with $0.5 \%$ Triton (TBS+, $\mathrm{pH} \mathrm{8.6)}$ ) for $72 \mathrm{~h}$ (at $4^{\circ} \mathrm{C}$ ). After rinsing, sections were incubated in biotinylated donkey anti-goat (List Biol. Lab.; 2 h). Following a final incubation with avidine-biotine complex (ABC Elite kit, Vector, Burlingame, $\mathrm{CA} ; 2 \mathrm{~h}$ ), they were processed with $\mathrm{DAB}$ for $30 \mathrm{~min}$. The WGA-BSA-gold label was enhanced by silver intensification (Aurion) resulting in black gold/silver particles located in labeled neurons which light up with darkfield illumination (Fig. 1B-D). CTb labeled neurons contained the brown DAB reaction product. Both labels could be easily identified within the same neuron (Fig. $1 \mathrm{~B}, \mathrm{E})[12]$. Of each case, labeled neurons were plotted in 

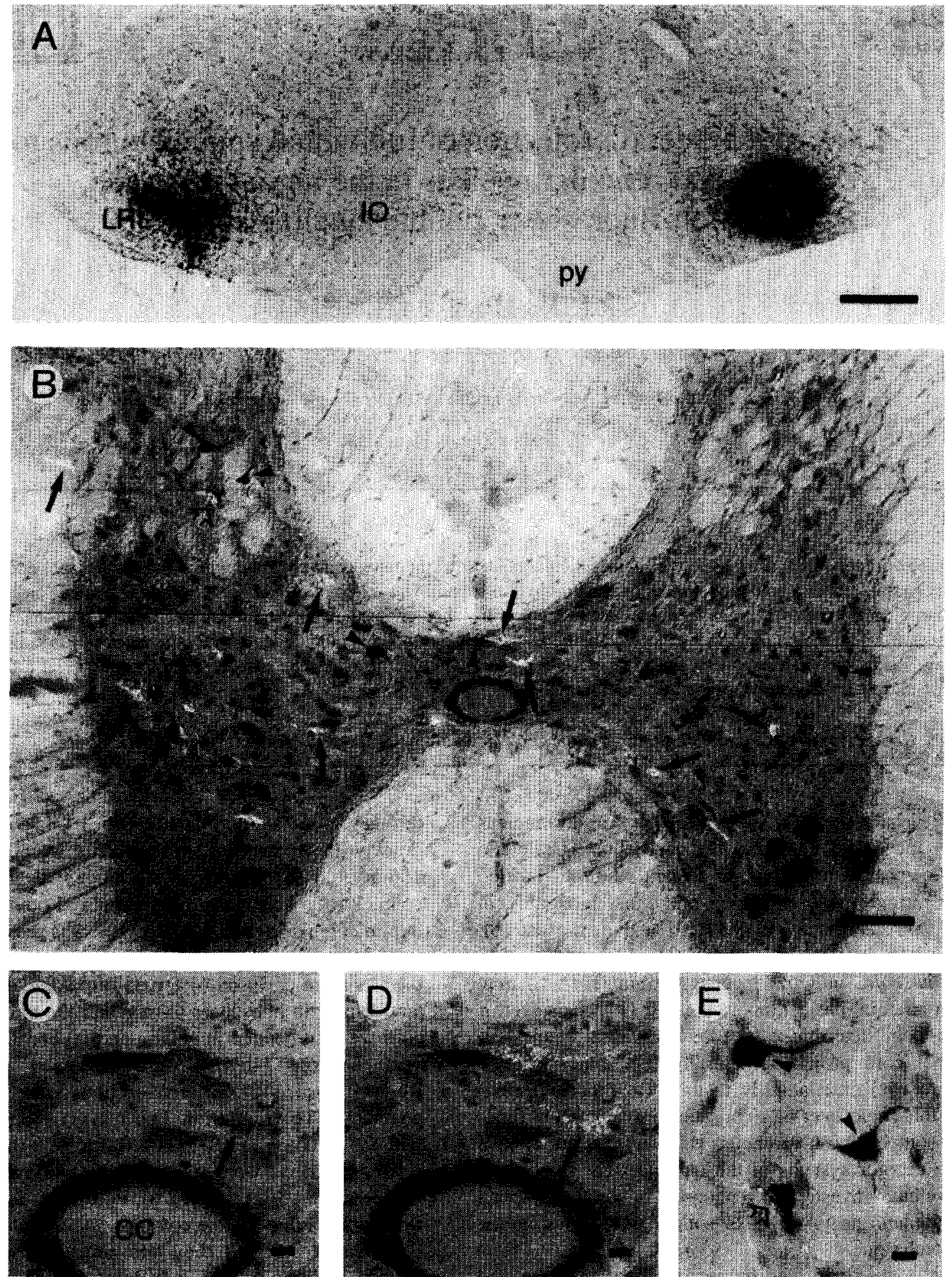

Fig. 1. Microphotographs related to case R528. (A) WGA-BSA-gold and CTb injection sites centered on the medial part of the left and right LRt, respectively. (B) Retrogradely labeled neurons in a thionin counterstained section from the caudal thoracic spinal cord. CTb labeled neurons appear as dark shapes (arrowheads) whereas gold labeled neurons light up (arrows). One double labeled neuron (open arrow) is seen in lamina IV/V of the left dorsal horn. Combined lightfield/darkfield illumination. (C) Detail of a CTb and a gold labeled neuron, located in lamina X of the section shown in (B). Lightfield illumination. (D) Same neurons visualized with a combination of lightfield and darkfield illumination. Note that the gold labeled neuron is easily recognized and that another neuron (arrow) that was out of focus in (C) now becomes visible also (cf. arrow in (C,D)). (E) Detail of a double labeled neuron (open arrow, also shown in (B)) as seen with combined lightfield/ darkfield illumination. Bars: $500 \mu \mathrm{m}$ in (A), $100 \mu \mathrm{m}$ in (B), and $10 \mu \mathrm{m}$ in (C-E). 

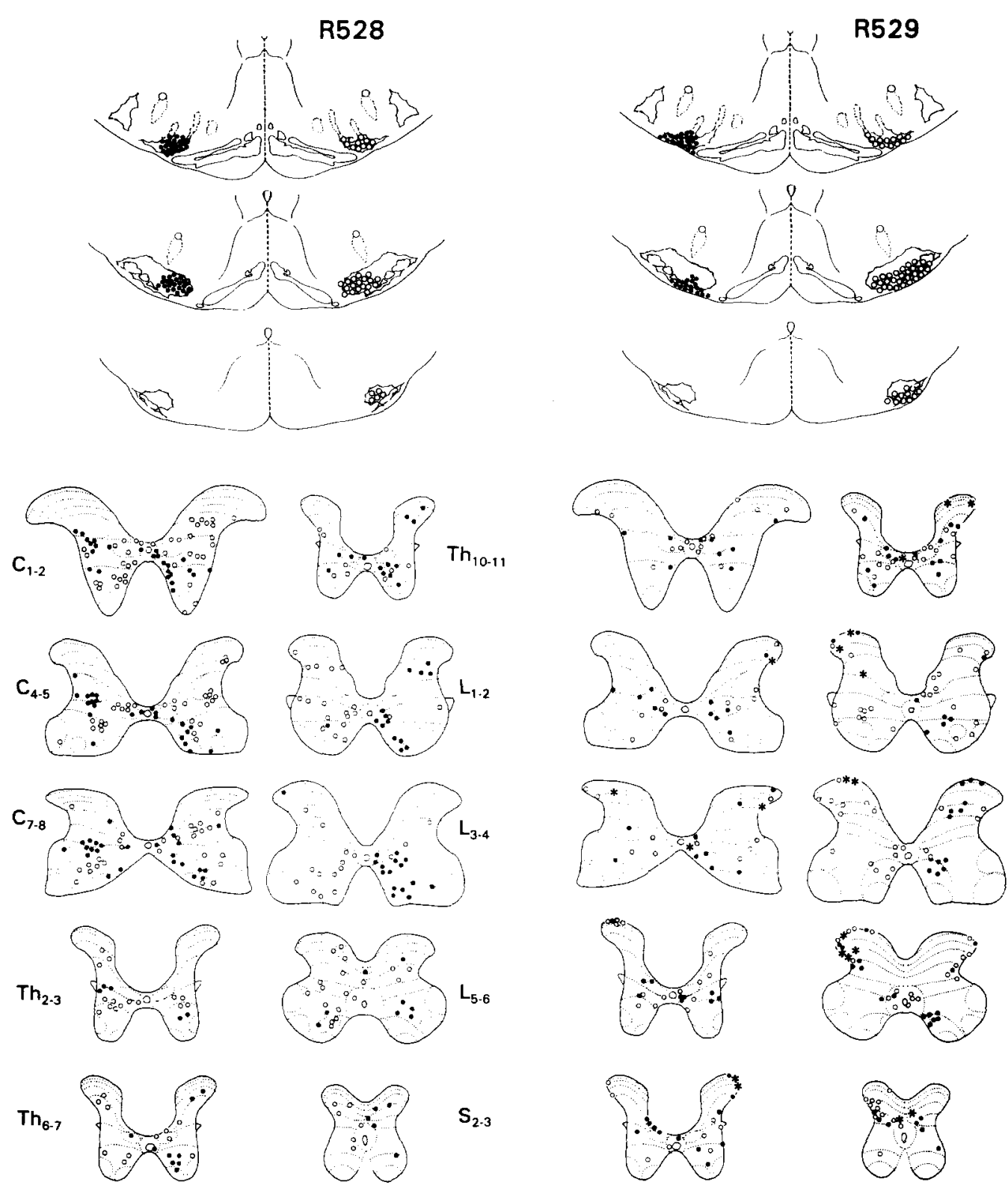

Fig. 2. Schematic diagram of the LRt injection sites (top panels) and resultant retrograde labeling in individual sections of various spinal cord levels (bottom panels) of cases R528 and R529. CTb injection site and labeled neurons are indicated by open circles, WGA-BSA-gold injection site and gold labeled neurons as black dots. Double labeled neurons are indicated with asterisks.

representative sections of three cervical $(\mathrm{C} 1-2, \mathrm{C} 4-5$ and C7-8), three thoracic (Th2-3, Th6-7 and Th10-11) and three lumbar (L1-2, L3-4 and L5-6) levels, and one sacral (S2-3) level.

Two cases with rather symmetrical injection sites will be described: R528 (Fig. 1) and R529. Their injections were centered on the medial part of the LRt but, in case R529, both injections were positioned more ventral compared to R528 (Fig. 2). The results of the other rats were quite similar to those of rat R528. Both cases resulted in numerous retrogradely labeled cells in the spinal cord (Figs. 1 and 2). At the cervical level, both gold and CTb labeled neurons were confined to lamina VIII contralat- erally, to laminae VII and X bilaterally, and to laminae III, IV and V ipsilaterally to their injection sites. At thoracic levels, most labeled neurons were located bilaterally within laminae VII, VIII and X and contralaterally within laminae IV and V. The labeling at lumbar levels was found mainly in laminae IV, V, and X bilaterally, and in laminae VII and VIII contralaterally to their injection sites (Fig. 2). The labeled sacral neurons were nearly all located in the intermediate gray matter. These results are essentially similar to those reported earlier $[9,13]$.

Case R529 differed from R528 in the sparsity of labeled neurons in the deep layers of the dorsal horn and adjacent intermediate gray of the ipsilateral cervical cord. 


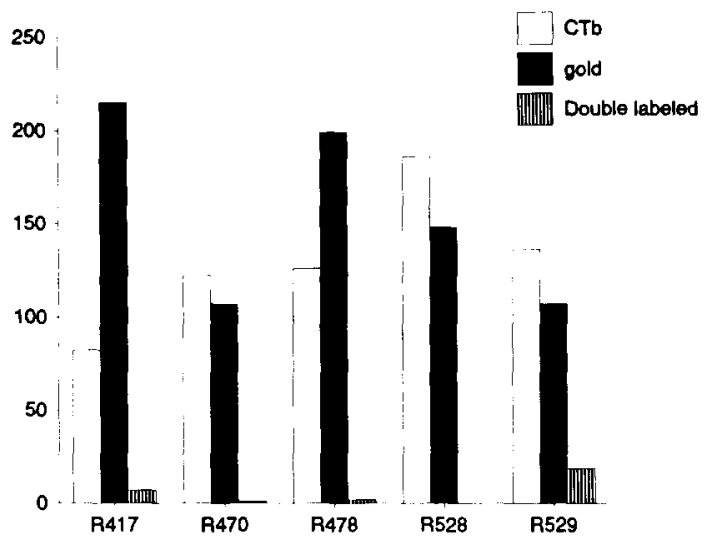

Fig. 3. Diagram indicating number of labeled neurons (ordinate) in ten sections of representative spinal cord levels of five different rats. Open bars, CTb labeled neurons; black bars, gold labeled neurons; hatched bars, double labeled neurons.

This is in accordance with the notion that these latter neurons project dorsomedially within the ipsilateral LRt and may represent the origin of the ipsilateral forelimb tract as described for the cat (for review, see [11]). In R529, moreover, labeled neurons were found within the superficial-most layers of the dorsal horn, especially at thoracic and lumbar levels.

Almost all labeled neurons contained either $\mathrm{CTb}$ or gold label. Double labeled neurons were rarely observed. In the 50 selected sections of all five rats, a total of 1457 labeled neurons was counted, 652 of them were CTb labeled and 776 were gold labeled (Fig. 3). Only 29 neurons contained both labels. Most of these (16) were found in case R529 and were frequently located in the superficial laminae of the dorsal horn. Since it has been shown that neurons located in these laminae project to areas adjacent to the LRt such as the ventrolateral reticular formation and the dorsal reticular nucleus [1,7], it is possible that labeled neurons in the superficial dorsal horn may project outside the LRt, especially since we used a dorsal approach for injecting the LRt. Another potential source for inadvertent labeling was the fact that in R529 the injection sites were close to the ventral surface of the brain stem where passing fibers may have taken up the tracers $[2,12]$. The actual number of neurons projecting to both LRts, therefore, may be overestimated. Hence, we conclude that most, if not all, spinal neurons with projections to the LRt direct their terminals either ipsi- or contralaterally.

Neurons in the LRt are reported to collateralize extensively to both sides of the cerebellar cortex where they may overlap with the mossy fiber projection from the LRt of the other side $[5,10]$. Also, many LRt neurons have been shown to respond to activation of the bilateral ventral flexor reflex tract [3], indicating that each LRt may be involved in movement control on both sides of the body. Nevertheless, our results suggest that information concerning the left and right limbs is distributed separately to both LRts. Recently, both excitatory and inhibitory monosynaptic spinal inputs to LRt neurons have been found [4] suggesting that it may be interesting to investigate the nature and origin of both types of spinoreticular connections and their relation to ipsilaterally or contralaterally projecting neurons.

The authors thank Mr. J. van der Burg and Mrs. H. Sabel-Goedknegt for their expert technical assistance.

[1] Almeida, A., Tavares, I., Lima, D. and Coimbra, A., Descending projections from the medullary dorsal reticular nucleus make synaptic contacts with spinal cord lamina I cells projecting to that nucleus: an electron microscopic tracer study, Neuroscience, 55 (1993) 1093-1106.

[2] Chen, S., Evidence that cholera toxin B subunit $(\mathrm{CTb})$ can be avidly taken up and transported by fibers of passage, Brain Res., 674 (1995) 107-111.

[3] Clendenin, M., Ekerot, C.-F., Oscarsson, O. and Rosén, I., The lateral reticular nucleus in the cat, II: organization of component activated from bilateral ventral flexor reflex tract (bVFRT), Exp. Brain Res., 21 (1974) 487-500.

[4] Ekerot, C.-F., The lateral reticular nucleus in the cat, VI: excitatory and inhibitory afferent paths, Exp. Brain Res., 79 (1990) 109-119.

[5] Ghazi, H., Hrycyshyn, A.W. and Flumerfelt, B.A., Doublelabeling study of axonal branching within the lateral reticulocerebellar projection in the rat, J. Comp. Neurol., 258 (1987) 378386.

[6] Hrycyshyn, A.W. and Flumerfelt, B.A., A light microscopic investigation of the afferent connections of the lateral reticular nucleus in the cat, J. Comp. Neurol., 197 (1981) 477-502.

[7] Lima, D. and Coimbra, A., Neurons in the substantia gelatinosa rolandi (lamina II) project to the caudal ventrolateral reticular formation of the medulla oblongata in the rat, Neurosci. Lett., 132 (1991) 16-18.

[8] Menétrey, D., Retrograde tracing of neural pathways with a protein-gold complex, I: light microscopic detection after silver intensification, Histochemistry, 83 (1985) 391-395.

[9] Menétrey, D., Roudier, F. and Besson, J.M., Spinal neurons reaching the lateral reticular nucleus as studied in the rat by retrograde transport of horseradish peroxidase, J. Comp. Neurol., 220 (1983) 439-452.

[10] Qvist, H., The cerebellar nuclear afferent and efferent connections with the lateral reticular nucleus in the cat as studied with retrograde transport of WGA-HRP, Anat. Embryol., 179 (1989) 471483.

[11] Ruigrok, T.J.H. and Cella, F., Precerebellar nuclei and red nucleus. In G. Paxinos (Ed.), Precerebellar Nuclei and Red Nucleus, Academic Press, Sydney, 1994, pp. 277-308.

[12] Ruigrok, T.J.H., Teune, T.M., van der Burg, J. and Sabel, H., A retrograde double labeling technique for light microscopy: a combination of axonal transport of cholera toxin B-subunit and a gold-lectin conjugate, J. Neurosci. Meth., (1995) in press.

[13] Shokunbi, M.T., Hrycyshyn, A.W. and Flumerfelt, B.A., Spinal projections to the lateral reticular nucleus in the rat: a retrograde labelling study using Horseradish peroxidase, J. Comp. Neurol., 239 (1985) 216-226. 